\title{
Tibial Tubercle Osteotomy in Total Knee Arthroplasty: Midterm Results Experience of a Monocentric Study
}

\author{
Stefano Biggi $i^{1,2}$ Stefano Divano $^{3}$ Riccardo Tedino ${ }^{1,2}$ Andrea Capuzzo ${ }^{1,2}$ Stefano Tornago ${ }^{2}$ \\ Andrea Camera ${ }^{1,2}$ \\ 1 U.O. Ortopedia, Clinica Città di Alessandria - Policlinico di Monza, \\ Alessandria, Italy \\ 2 Fondazione Lorenzo Spotorno - ONLUS, Albenga, Italia \\ ${ }^{3}$ Clinica Ortopedica e Traumatologica, Ospedale Policlinico San \\ Martino, Genova, Italy \\ Joints 2018;6:95-99.

\begin{abstract}
Address for correspondence Stefano Biggi, MD, U.O. Ortopedia, Clinica Città di Alessandria-Policlinico di Monza, Via Moccagatta 30,
\end{abstract} \\ 15121 Alessandria (AL), Italy (e-mail: dott.sbiggi@gmail.com).
}

\begin{abstract}
Keywords

- tibial tubercle osteotomy

- total knee arthroplasty

- revision

- surgical approach

Purpose Difficult primary total knee arthroplasty (TKA) and revision TKA may be high demanding, especially during joint exposure. Aim of this article is to evaluate the clinical and radiological outcomes of a series of patients, who underwent TKA and revision TKA, where tibial tubercle osteotomy (TTO) was performed.

Methods We retrospectively reviewed a cohort of 79 consecutives TKAs where TTO was performed. Patients were assessed clinically and radiographically at their last follow-up (mean, $7.4 \pm 3.7$ years). Clinical evaluation included the Knee Society Score (KSS), the pain visual analogue scale (VAS), and range of motion. Radiological assessment included the evaluation of radiolucent lines, osteolysis, cortical bone hypertrophy, time of bone healing of the TTO fragment, and the hardware complication.

Results KSS raised from $40.7 \pm 3.1$ to $75 \pm 4.3(p<0.0001)$. Knee flexion increased from $78.7 \pm 9.9^{\circ}$ to $95.0 \pm 9.5^{\circ}(p<0.0001)$, and VAS improved from $7.9 \pm 0.9$ to $3.8 \pm 1(p<0.0001)$. No signs of loosening or evolutive radiolucency lines were found. Osteolytic areas around the stem were detected. No significant association was found between the implant design and the outcomes, while aseptic loosening showed significantly better results. Complications were: 4 painful hardware, 3 late periprosthetic infections, 1 extension lag of $5^{\circ}$, and 3 flexion lag.

Conclusion Our experience suggests the use of TTO to improve the surgical approach in difficult primary TKA or revision TKA. A precise surgical technique leads to good results with low risk of complications.

Level of Evidence Level IV, therapeutic case series.
\end{abstract}

\section{Introduction}

Tibial tubercle osteotomy (TTO) is a well-established surgical procedure to improve the surgical approach during revision total knee arthroplasty (TKA) or difficult primary TKA. First described by Dolin ${ }^{1}$ and later by Wolff et al, ${ }^{2}$ TTO owes its popularity and diffusion to Whiteside and $\mathrm{Ohl}^{3}{ }^{3}$ who standardized the surgical technique and reported good outcomes. $^{4}$

The extensile approach described by these authors consists in the osteotomy of a fragment containing the anterior tibial tuberosity and the patellar tendon, approximately 7 to $11 \mathrm{~cm}$ long and at least $2 \mathrm{~cm}$ thick; the fragment is therefore retracted laterally, and the fully knee exposure is achieved. If received

October 2, 2017

accepted after revision

May 20, 2018

published online

June 22, 2018
DOI https://doi.org/

10.1055/s-0038-1661338. ISSN 2282-4324.
Copyright (c) 2018 Georg Thieme Verlag License terms

KG Stuttgart · New York 
performing a lateral parapatellar approach, the bone fragment can be medially retracted.

Indications for TTO are: (1) the need for an adequate joint visualization when standard approaches are not sufficient; (2) the inability to dislocate the patella at $90^{\circ}$ of flexion without the risk of patellar tendon rupture; (3) the need to access the tibial canal; and (4) the correction of a concomitant patella baja. ${ }^{5}$ Relative contraindication for TTO is a massive tibial osteolysis that could jeopardize the fragment fixation. ${ }^{6}$ The use of TTO during a two-stage procedure, or repeated TTO, is not considered as contraindications. ${ }^{7}$

The purpose of this article is to evaluate, retrospectively, the results of primary or revision TKAs where TTO was performed. The hypothesis of the study was that the TTO is a safe and effective approach in TKA.

\section{Methods}

Using the Institution Surgical Registry, we identified all of the TKAs or revision TKAs in which TTO was performed from 2000 to 2014.

The surgical approach, as described by Whiteside and $\mathrm{Ohl}^{3}$ and used by the authors, consists of distal extension of the skin incision of approximately 10 to $12 \mathrm{~cm}$; the dissection continues in line with the skin incision with a subperiosteal flap in the medial soft tissues, and then the anterior aspect of the tibia is exposed. A ruler is used to mark the length of the osteotomy at the planned level; usually the fragment should be approximately 7 to $11 \mathrm{~cm}$ long and at least $2 \mathrm{~cm}$ thick to ensure the bone healing of the fragment. The medial tibial cortex is divided using an oscillating saw. Then, the osteotomy of the anterior tibial cortex is extended distally. Using multiple broad osteotomes, the lateral tibial cortex is divided with as much uniformity as possible to prevent fragmentation of the osteotomized tibia. With the osteotomes engaged in the lateral tibial cortex, they are elevated as a unit, and the osteotomy fragment is hinged on the lateral soft tissues, taking care to avoid and minimize disruption of the lateral muscular attachments to the fragment. The entire knee joint is then visualized. It is extremely important that the lateral soft tissues are preserved and attached to the osteotomy fragment to maintain vascularization, avoiding fractures and nonunions (-Fig. 1). After the components' revision, the synthesis of the bone fragment is performed with two divergent $3.5-\mathrm{mm}$ diameter cortical screws and the knee flexed at $90^{\circ}$. This position allows the correct patellar centering, reestablishing a correct joint line and good range of motion (ROM) (-Fig. 2).

Clinical and radiographic evaluations were performed at 45 days, 3, 6, and 12 months after surgery, and then annually.

Clinical assessment included the Knee Society Score (KSS) and the pain visual analogue scale (VAS). The KSS questionnaire used in this study included the 1993 modifications recommended by Insall et al. ${ }^{8}$ ROM was evaluated with a hand-held goniometer.

Radiographic assessment was performed with standard anteroposterior (AP) and lateral knee X-rays and included the evaluation of radiolucent lines, osteolysis, cortical bone

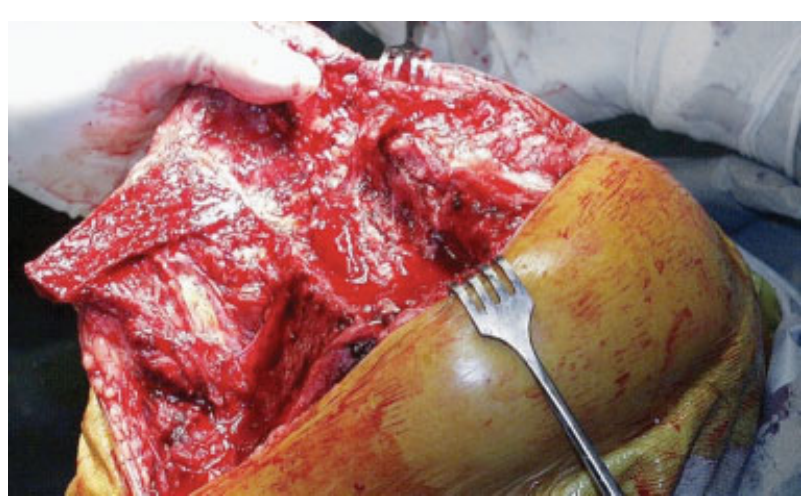

Fig. 1 Intraoperative picture of knee exposure after tibial tubercle osteotomy (TTO) and prosthetic components removal. The lateral soft tissues are preserved to maintain vascularization.

hypertrophy, the time of bone healing of the TTO fragment, and any hardware complication (i.e., screw breakage, pullout, or loosening) (-Fig. 3). The osteolytic areas were quantified by using the maximum diameter and the widest length perpendicular to this diameter. The areas were approximated an ellipses. ${ }^{9}$ The osteolytic areas were classified as $\leq 1 \mathrm{~mm}^{2}, 1$ to $5 \mathrm{~mm}^{2}$, and $>5 \mathrm{~mm}^{2}$. Radiological findings were described using the areas of Whaley et $\mathrm{al}^{10}$ (-Fig. 4).

For statistical analysis, categorical data were recorded with frequencies and percentages, while continuous data with means and standard deviations. Comparisons between groups were performed by the Student's $t$-test for continuously distributed data, and for proportions with the chisquare test or Fisher's exact test as appropriate, considering the value of $p<0.05$ as statistically significant.

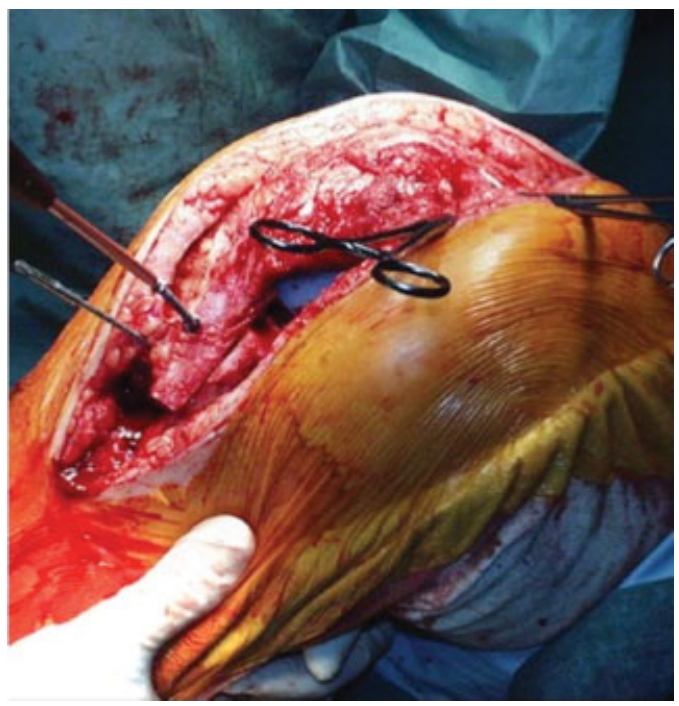

Fig. 2 Fixation of the bone fragment is performed using two divergent 3.5-mm diameter cortical screws with the knee flexed at $90^{\circ}$, thus allowing the correct patellar centering and reestablishing a correct joint line. 


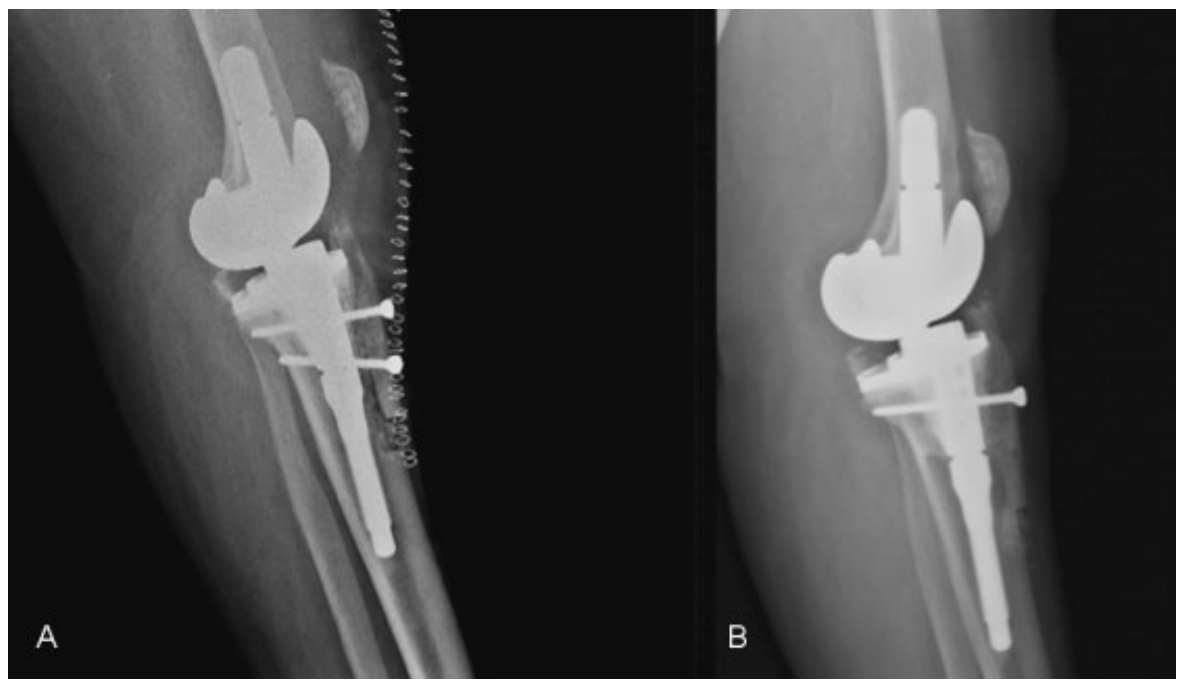

Fig. 3 (A) Postoperative lateral X-ray showing revision total knee arthroplasty using tibial tubercle osteotomy, and subsequent fixation with two screws. Patella achieves the correct position. (B) Lateral X-ray at 2-year follow-up shows fragment healing. One screw has been removed.

\section{Results}

The entire cohort included 79 patients, 21 men (25\%) and 58 women $(75 \%)$. The mean age at surgery was $73.6 \pm 3$ years (range, 49-78 years). Preoperative diagnosis was aseptic loosening in 22 cases (27.8\%), septic two-stage revision in 54 cases $(68.4 \%)$, one case (3.8\%) of primary osteoarthritis
(OA) with patella baja, and one case (3.8\%) of posttraumatic OA with extension and flexion lag, patella baja, and articular stiffness following primary TKA.

With regard to the prosthetic design, a constrained condylar knee (CCK) prosthesis (NexGen LCCK; Zimmer Inc., Warsaw, Indiana, United States) was implanted in 67 cases, while a rotating hinge $(\mathrm{RH})$ prosthesis (NexGen RHK,
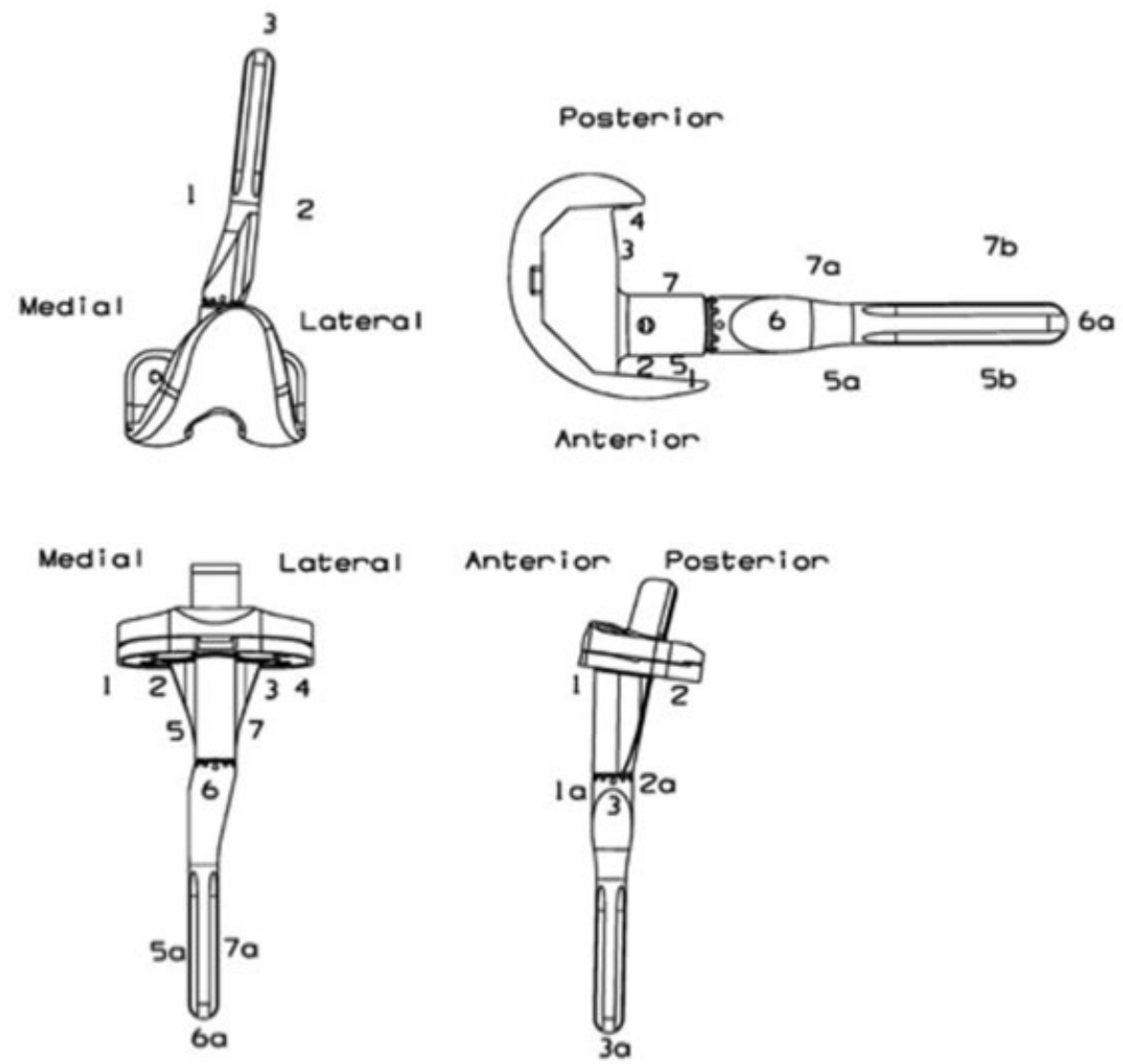

Fig. 4 Radiographic zones according to Whaley et al. ${ }^{10}$ 
Zimmer) was implanted in 9 cases. In the case of primary OA, a first implant posterior-stabilized design was implanted (NexGen LPS, Zimmer). For the patient with articular stiffness no implant revision was performed.

Mean preoperative KSS was $40.7 \pm 3.09$ points (range, 35-55). Mean preoperative VAS was $7.9 \pm 0.9$ (range, 6-9). Mean preoperative knee flexion was $78.7 \pm 9.9^{\circ}$ (range, 5090). Concerning knee extension, 9 cases ( 6 two-stage revision, 1 aseptic loosening, the case of primary $O A$, and the patient complaining knee stiffness) showed an extension lag of $10^{\circ}$, while 13 cases (10 two-stage revision and 3 aseptic loosening) had an extension lag of $5^{\circ}$.

Mean follow-up time was $7.4 \pm 3.7$ years (range, 4-13 years). All the clinical outcomes significantly improved at follow-up: mean KSS was $75 \pm 4.3$ points (range, 66-90) $(p<0.0001)$; VAS was $3.8 \pm 1$ (range, $1-5)(p<0.0001)$; and knee flexion was $95 \pm 9.5^{\circ}$ (range, $\left.50-120\right)(p<0.0001)$. We found only one extension lag of $5^{\circ}$, whereas we reported 3 flexion lag (flexion $<80^{\circ}$ ); all 4 patients have undergone a twostage revision.

No significant association was found between the implant design (CCK or RH) and KSS ( $p=0.6$ ), degrees of flexion $(p=0.8)$, and VAS score $(p=0.8)$. Conversely, in preoperative diagnosis we found a significant positive association between aseptic loosening and KSS $(p<0.00001)$, flexion degrees $(p=0.04)$, and VAS $(p<0.0003)$.

On radiographic assessment, bone healing of the fragment was achieved in an average of $2.3 \pm 0.6$ months (range, 1.5-4) after surgery. We found a significant positive association between a quicker fragment healing and the preoperative diagnosis of aseptic loosening $(p<0.007)$, while no correlation was found between bone healing and implant design $(p=0.3)$. No sign of loosening or radiolucent lines were reported. In 8 cases (10\%), we found osteolytic areas between 1 and $5 \mathrm{~mm}^{2}$ around the stem. The overall involved areas according to Whaley et al ${ }^{10}$ were femoral AP 2, femoral lateral view 7, 5, 5a, and 5b, tibial AP 5, 5a, 6a, 7, 7a, and tibial lateral view 1a, 2a, and 3a. No significant association was found between the presence of osteolytic areas and implant design $(p=0.86)$, or preoperative diagnosis $(p=0.57)$.

We detected four cortical bone hypertrophies on 7a area, nonevolutive over 18 months, with slight pain at the tip of the stem.

In four cases (5\%), the screws were removed due to anterior pain, resulting in pain relief. No additional hardware complications were detected.

Further complications were 3 (4\%) late periprosthetic infections, 1 of them treated with antibiotic suppression, while the other 2 cases were treated with a further two-stage revision.

\section{Discussion}

Several approaches are described for TKA. ${ }^{11}$ Extensile approaches during difficult primary or revision TKA are used to release the stress on the extensor mechanism. In fact, postoperative surgical scars and fibrotic retraction of the patellar tendon make it less elastic and more prone to breakage. In general, when it is impossible to dislocate the patella at $90^{\circ}$ of flexion without the risk of a patellar tendon rupture, an extensile approach is required. ${ }^{12}$

Starting from a standard medial parapatellar approach, several methods are described to improve the exposure of the knee joint protecting the extensor mechanism integrity, such as the Tarabichi's maneuver, ${ }^{13}$ the extensor mechanism tenolysis, ${ }^{14}$ the "banana peel," ${ }^{15}$ or the Insall's quadriceps snip. ${ }^{16}$ Further extensile approach are quadriceps turndown or TTO.

Quadriceps turndown, evolved from the original description of Coonse and Adams ${ }^{17}$ to Scott and Silliski's modification, ${ }^{18}$ leads to a proximal interruption of the quadriceps tendon and a distal turndown of the patella and the patellar tendon. Articular visualization is excellent as well as recovery of the flexion, but there are several pitfalls. First, the quadriceps detachment area is wide and, despite a good reconstruction, it may results to an important extension lag. Moreover, the proximity of the superolateral genicular artery leads to an increased risk of bleeding or osteonecrotic lesions of the patella, apparently asymptomatic as reported by Smith et al. $^{19}$

Conversely, TTO consists in a distal interruption of the extensor mechanism, where an osteoperiosteal flap containing the anterior tibial tubercle and the patellar tendon is laterally retracted. ${ }^{20}$ This procedure presents different advantages: first, quadriceps muscle is spared, patella baja can be corrected, and the wide exposure leads to direct survey of the tibial canal. ${ }^{21,22}$ On the other hand, its use limited to particularly difficult cases involves the theoretical onset of various complications, including mechanical failures, delayed healing, or nonunion of the fragment, proximal migration of the fragment, wound complications, stiffness, extension lag, or loss of flexion. ${ }^{23,24}$

We performed the fixation of the fragment using two screws, according to Davis et $\mathrm{al}^{24}$ without detecting any hardware complications or proximal migration of bone fragment.

Several authors reported low complication rates concerning the hardware or the fragment fixation if a stable fixation is achieved. ${ }^{4-7,23}$

No significant association was found between the type of prosthetic implant and the clinical or radiological parameters. Conversely, aseptic loosening showed significant association with higher postoperative KSS, greater postoperative knee flexion, improvement in VAS, and lower time of fragment healing. These findings could be explained as periarticular soft tissues in aseptic loosening have better quality than in periprosthetic infections, and this allows for better postoperative rehabilitation and faster bone healing. Probably, additional drug therapies in a septic condition may affect the outcomes.

Osteolytic areas were reported at follow-up. Osteolysis was observed in the same areas for most patients, in the absence of further signs of loosening or evolutive radiolucent lines. Probably, these findings are the result of a stressshielding effect of the implant, and a redistribution of loads due to the presence of the stem. 
The study has some relevant limitations. First, its retrospective nature impairs external validity of the results. Second, follow-up time was not homogeneous within the study population and no longitudinal data were available. Finally, no control group was available.

In conclusion, TTO is a valid and safe option in difficult primary and revision TKA. Following correct indications and performing a precise surgical technique, TTO is a reliable option during joint exposure in difficult cases, with low complications rate. However, further studies with larger groups of patients and a longer follow-up are needed to better evaluate the outcomes and safety of TTO.

\section{Conflict of Interest}

None.

\section{References}

1 Dolin MG. Osteotomy of the tibial tubercle in total knee replacement. A technical note. J Bone Joint Surg Am 1983;65(05): 704-706

2 Wolff AM, Hungerford DS, Krackow KA, Jacobs MA. Osteotomy of the tibial tubercle during total knee replacement. A report of twenty-six cases. J Bone Joint Surg Am 1989;71(06):848-852

3 Whiteside LA, Ohl MD. Tibial tubercle osteotomy for exposure of the difficult total knee arthroplasty. Clin Orthop Relat Res 1990; (260):6-9

4 Whiteside LA. Exposure in difficult total knee arthroplasty using tibial tubercle osteotomy. Clin Orthop Relat Res 1995;(321): 32-35

5 Mendes MW, Caldwell P, Jiranek WA. The results of tibial tubercle osteotomy for revision total knee arthroplasty. J Arthroplasty 2004;19(02):167-174

6 Ries MD, Richman JA. Extended tibial tubercle osteotomy in total knee arthroplasty. J Arthroplasty 1996;11(08):964-967

7 Chalidis BE, Ries MD. Does repeat tibial tubercle osteotomy or intramedullary extension affect the union rate in revision total knee arthroplasty? A retrospective study of 74 patients. Acta Orthop 2009;80(04):426-431

8 Insall JN, Dorr LD, Scott RD, Scott WN. Rationale of the Knee Society clinical rating system. Clin Orthop Relat Res 1989;(248): $13-14$
9 Zicat B, Engh CA, Gokcen E. Patterns of osteolysis around total hip components inserted with and without cement. J Bone Joint Surg Am 1995;77(03):432-439

10 Whaley AL, Trousdale RT, Rand JA, Hanssen AD. Cemented longstem revision total knee arthroplasty. J Arthroplasty 2003;18(05): 592-599

11 Abbott LC, Carpenter WF. Surgical approaches to the knee joint. J Bone Joint Surg Am 1945;27:277-310

12 Younger AS, Duncan CP, Masri BA. Surgical exposures in revision total knee arthroplasty. J Am Acad Orthop Surg 1998;6(01):55-64

13 Tarabichi S, Tarabichi Y. Can an anterior quadriceps release improve range of motion in the stiff arthritic knee? J Arthroplasty 2010;25(04):571-575

14 Sharkey PF, Homesley HD, Shastri S, Jacoby SM, Hozack WJ, Rothman RH. Results of revision total knee arthroplasty after exposure of the knee with extensor mechanism tenolysis. J Arthroplasty 2004;19(06):751-756

15 Lahav A, Hofmann AA. The "banana peel" exposure method in revision total knee arthroplasty. Am J Orthop 2007;36(10): 526-529

16 Garvin KL, Scuderi G, Insall JN. Evolution of the quadriceps snip. Clin Orthop Relat Res 1995;(321):131-137

17 Coonse KD, Adams JD. A new operative approach to the knee joint. Surg Gynecol Obstet 1943;77:344-347

18 Scott RD, Siliski JM. The use of a modified V-Y quadricepsplasty during total knee replacement to gain exposure and improve flexion in the ankylosed knee. Orthopedics 1985;8(01):45-48

19 Smith PN, Parker DA, Gelinas J, Rorabeck CH, Bourne RB. Radiographic changes in the patella following quadriceps turndown for revision total knee arthroplasty. J Arthroplasty 2004;19(06):714-719

20 van den Broek CM, van Hellemondt GG, Jacobs WC, Wymenga AB. Step-cut tibial tubercle osteotomy for access in revision total knee replacement. Knee 2006;13(06):430-434

21 Halder AM. Tibial tubercle osteotomy [in German]. Oper Orthop Traumatol 2012;24(02):85-94

22 Barrack RL, Smith P, Munn B, Engh G, Rorabeck C. The Ranawat Award. Comparison of surgical approaches in total knee arthroplasty. Clin Orthop Relat Res 1998;(356):16-21

23 Young $\mathrm{CF}$, Bourne RB, Rorabeck $\mathrm{CH}$. Tibial tubercle osteotomy in total knee arthroplasty surgery. J Arthroplasty 2008;23(03): 371-375

24 Davis K, Caldwell P, Wayne J, Jiranek WA. Mechanical comparison of fixation techniques for the tibial tubercle osteotomy. Clin Orthop Relat Res 2000;(380):241-249 